\title{
Implementación de una lista de cotejo para mejorar la adherencia a prácticas basadas en evidencia en una Unidad de Cuidados Intensivos Pediátricos
}

\author{
Implementation of a checklist to increase adherence to \\ evidence-based practices in a single pediatric intensive care unit
}

\author{
Dr. Pablo Eulmesekian ${ }^{a}$, Méd. Augusto Pérez ${ }^{a}$ Méd. Silvia Díaz y Méd. Mateo Ferrero ${ }^{a}$
}

\section{RESUMEN}

Introducción. Eluso delistas de cotejo para mejorar la adherencia a prácticas basadas en evidencia en unidadesdecuidadosintensivospediátricosnoestá generalizado. Elobjetivodelestudiofue, mediante una lista específicamentediseñada, alcanzare190\% de adherencia a las prácticas estudiadas.

Población y métodos. Estudio cuasiexperimental tipo serie de tiempo en niños ventilados en la Unidad de Cuidados Intensivos Pediátricos. Las prácticas estudiadas fueron ventana de sedación, presión plateau $\leq 30 \mathrm{cmH}_{2} \mathrm{O}$, fracción inspirada de oxígeno $\leq 60 \%$, cabecera a $30^{\circ}$, higiene bucal con clorhexidina, recambio semanal del circuito del respirador, preferencia de alimentación enteral, disminución delumbral de transfusiones (hemoglobina: $7 \mathrm{~g} / \mathrm{dl}$ ), consideración diaria de prueba de respiración espontánea y de retiro de catéter central. La lista fue utilizada durante el pase de sala, por médicos de planta responsables de la Unidad de Cuidados Intensivos Pediátricos, como intervención para mejorar la adherencia y herramienta de registro. Se consideró observación a cada formulario completado diariamente. Las observaciones fueron clasificadas como defectuosas si no hubo adherencia a uno o más ítems. La adherencia (proporción de observaciones sin defecto) se resume en el gráfico de control.

Resultados. El estudio abarcó 420 días. Se internaron732 pacientes; 218 recibieronventilación mecánica; se realizaron 1201 observaciones y 1191 fueron incluidas. El gráfico de control con horizonte temporal de 14 meses mostró un aumentodeadherencia, un patrón devariabilidad de causa especial en los últimos 3 meses y adherencia $>90 \%$ en los últimos dos.

Conclusiones. El uso de la lista de cotejo permitió mejorar la adherencia a las prácticas estudiadas y alcanzar más de $90 \%$ en los últimos 2 meses. Palabras clave: lista deverificación, prácticabasada en laevidencia, cuidados intensivos, pediatría, calidad de la atención de salud.

http: / / dx.doi.org/10.5546/ aap.2017.446

Texto completo en inglés:

http: / / dx.doi.org/10.5546/ aap.2017.eng.446

Ninguno.

Conflicto de intereses: Ninguno que declarar.

Recibido: 5-12-2016 Aceptado: 17-4-2017 Cómo citar: Eulmesekian P, Pérez A, Díaz S, et al. Implementación de una lista de cotejo para mejorar la adherencia a prácticas basadas en evidencia en una Unidad de Cuidados Intensivos Pediátricos. Arch Argent Pediatr 2017;115(5):446-452.

\section{INTRODUCCIÓN}

El Institute of Medicine, EE. UU. define "calidad de atención de la salud" como el grado en que los servicios de salud aumentan la probabilidad de obtener resultados deseados consistentes con un conocimiento científico actualizado, por lo tanto, existe relación entre este concepto y la adherencia a prácticas basadas en evidencia. ${ }^{1}$ No obstante, la adherencia a prácticas que mejoran resultados en Terapia Intensiva es inconsistente. Algunos pacientes, por diferentes circunstancias, no se benefician de su aplicación. ${ }^{2-4}$

Pronovost estudió el impacto de no cumplir tratamientos que mejoraban la sobrevida de adultos internados en Terapia Intensiva y estimó que se podrían salvar casi 170000 vidas por año si se cumplieran las prácticas basadas en evidencia estudiadas. ${ }^{5}$

Las listas de verificación contribuyen a evitar errores de omisión y constituyen una herramienta útil para completar en forma segura desde tareas simples, hacer las compras, hasta complejas, pilotear un avión. ${ }^{6}$ Su uso se ha extendido a diferentes áreas de la medicina, por ejemplo, para diagnosticar muerte cerebral, ${ }^{7}$ mejorar la adherencia a tratamientos de infarto miocárdico y accidente cerebrovascular ${ }^{8}$ o contribuir a disminuir la mortalidad intraquirúrgica. ${ }^{9}$

Aunque el uso de listas de verificación en Terapia Intensiva se ha difundido, no está generalizado. En adultos, se las utiliza para mejorar la comunicación, el cumplimiento de 
objetivos $^{10}$ y como herramienta para mejorar la adherencia a prácticas basadas en evidencia. ${ }^{11-15}$ En Cuidados Intensivos Pediátricos, existen menos referencias sobre su utilización. Se las ha empleado para mejorar la comunicación de objetivos asistenciales ${ }^{16}$ y para optimizar el cumplimiento de prácticas relacionadas con la seguridad del paciente. ${ }^{17}$ En nuestro medio, su uso no ha sido reportado.

El objetivo principal del estudio fue, mediante el uso de una lista de cotejo específicamente diseñada, alcanzar $90 \%$ de adherencia a prácticas basadas en evidencia en niños ventilados en la Unidad de Cuidados Intensivos Pediátricos (UCIP) del Hospital Italiano de Buenos Aires (HIBA). Los objetivos secundarios fueron evaluar la evolución de dicha adherencia durante los meses del estudio e identificar las prácticas con menor y mayor porcentaje de adherencia.

\section{POBLACIÓN Y MÉTODOS Ámbito}

La UCIP del HIBA es una unidad multidisciplinaria de 20 camas, con un promedio de 750 admisiones anuales y una tasa de uso de asistencia respiratoria mecánica (ARM) de $40 \%$. Se encuentra inserta en un hospital general universitario acreditado por Joint Commission International.

El pase de sala es una actividad central. Se realiza todas las mañanas y participan profesionales de diferentes especialidades. Cuatro médicos de planta rotan semanalmente para dirigir la Unidad y liderar esta actividad. Durante ella, se toman decisiones diagnósticas, terapéuticas y se definen objetivos asistenciales.

\section{Diseño, población y período de estudio}

El estudio correspondió a un diseño cuasiexperimental tipo serie de tiempo. Su horizonte temporal fue de 12 meses más el período de prueba piloto y fue realizado entre el $12 / 8 / 2013$ y el 5/10/2014. La población incluida fueron los pacientes con ARM internados en la UCIP. Los mayores de 18 años y traqueostomizados sin recibir sedantes en infusión endovenosa continua fueron excluidos.

\section{Prácticas estudiadas}

Las prácticas basadas en evidencia incluidas en la lista fueron las siguientes:

- Interrupción diaria de sedación. ${ }^{18,19}$

- Estrategia de protección pulmonar (presión plateau $\leq 30 \mathrm{cmH}_{2} \mathrm{O}$ y fracción inspirada de oxígeno $\left.-\mathrm{FIO}_{2}-\leq 60\right) .{ }^{20}$

- Medidas de prevención de neumonía asociada a respirador: elevación de la cabecera de la cama a más de $30^{\circ}$, higiene bucal con clorhexidina $0,12 \%$ cada 8 horas y recambio del circuito de respirador cada 7 días, siguiendo las recomendaciones del fabricante. ${ }^{21-23}$

- Consideración diaria de retiro del respirador, evaluando si el paciente se encontraba en condiciones de realizar una prueba de respiración espontánea. ${ }^{24,25}$

- Consideración diaria de retiro del catéter central. ${ }^{14,15,26}$

- Preferencia de alimentación enteral..$^{27,28}$

- Disminución del umbral de transfusiones de rojos a un valor de hemoglobina de $7 \mathrm{~g} / \mathrm{dl} .{ }^{29,30}$

- Planteamiento de objetivos claros durante el pase de sala. ${ }^{10}$

- Revisión de la medicación. ${ }^{31,32}$

\section{Etapas del estudio}

Diseño de la lista de cotejo

Se diseñó un formulario con datos demográficos del paciente, las prácticas mencionadas y un espacio para registrar razones de no adherencia. Los 4 médicos de planta, responsables de la Unidad y del uso de la lista, revisaron este formulario inicial.

\section{Prueba piloto y análisis periódicos}

La prueba piloto de la lista se realizó entre el 12 / 8 / 2013 y el 21/9/2013. Se identificaron problemas de diseño en el formulario y se realizaron modificaciones (véase Anexo en formato electrónico). Luego se realizaron 5 análisis bimestrales antes del análisis final. En cada uno, se verificaba el porcentaje de adherencia y se identificaban los ítems con menor grado de cumplimiento. Esta información era presentada por el autor principal del trabajo a los médicos que utilizaban la lista para mantener el compromiso con su uso.

\section{Uso de la lista de cotejo}

La lista se utilizó durante el pase de sala en todos los pacientes ventilados. Luego de la presentación del residente y de la definición de los objetivos del día, el médico de planta a cargo de la Unidad durante la semana revisaba en voz alta cada ítem y registraba su cumplimiento. Si alguno no se había cumplido, prescribía su cumplimiento por contar con los privilegios necesarios.

Ítems no cumplidos

Para definir que no se había adherido a una 
práctica, se identificaban los ítems marcados como no cumplidos y se observaba la existencia de alguna justificación. Si existía una explicación, el ítem se consideraba cumplido; por ejemplo, si un paciente se encontraba en decúbito prono, quien llenaba el formulario aclaraba junto al ítem "Cabecera de la cama a más de $30^{\circ "}$ que no era posible cumplir esta práctica por la posición del paciente. Por el contrario, si quien llenaba la lista omitía justificar la falta de adherencia, la práctica se consideraba no cumplida. La presencia de, al menos, un ítem no cumplido determinaba que la observación fuera considerada defectuosa. Los motivos de no cumplimiento no fueron estandarizados.

\section{Definición de variables}

- Unidad de observación: cada formulario llenado durante el pase de sala en cada paciente ventilado.

- Ítems de la unidad de observación: cada práctica incluida en la lista.

- Ítems cumplidos: aquellos efectivamente cumplidos o aquellos en los que la razón de no cumplimiento fue debidamente justificada.

- Ítems no cumplidos: aquellos marcados como no realizados sin justificación o sin una justificación adecuada.

- Unidad de observación defectuosa: aquella en la que, al menos, uno de sus ítems no fue cumplido.

- Unidad de observación sin defectos: aquella en la que todos sus ítems fueron categorizados como cumplidos.

- Proporción de unidades defectuosas: cociente entre el número de unidades defectuosas y el número de observaciones.

- Proporción de unidades sin defectos (indicador de adherencia): cociente entre el número de unidades sin defectos y el número de observaciones.

\section{Indicadores de resultado}

En forma simultánea, se registraron mortalidad, tasa de uso de ARM y catéter venoso central, tasa de neumonías asociadas a respirador y bacteriemias asociadas a catéter venoso central, porcentaje de errores en la prescripción médica y episodios de caídas.

\section{Análisis estadístico}

Como medidas de resumen, se utilizaron medias y desvíos estándar (DE) para variables con distribución normal; mediana y rango intercuartilos para aquellas con distribución no normal y proporciones para variables categóricas.

Para analizar el porcentaje de adherencia a las prácticas estudiadas en función del tiempo, se utilizaron gráficos de control. Estos permiten diferenciar variabilidad de causa común, aquella que sucede por azar, de variabilidad de causa especial. La distribución de puntos del gráfico establece esta diferenciación. Un patrón consistente en 1 punto fuera de los límites de control, 6 puntos sucesivos que aumentan o disminuyen $\mathrm{u} 8$ puntos sucesivos del mismo lado de la media define variabilidad de causa especial. Cuando estos gráficos reflejan la existencia de variabilidad de causa especial, es posible inferir que existe una probabilidad muy baja de que los cambios hayan sucedido por azar. Si, para límites de control del gráfico, se usan $\pm 3 \mathrm{DE}$, el error tipo I es de 0,0027 para cada medición y de $\left(1-0,9973^{\text {nediciones }}\right)$ para todo el gráfico..$^{33,34}$

En este estudio, se utilizó un gráfico tipo $\mathrm{P}$ con mediciones bisemanales y un horizonte temporal de 14 meses. Los límites de control se establecieron en \pm 3 DE. El análisis estadístico se realizó con Stata 9 (StataCorp, Texas).

\section{Aspectos éticos}

Los datos obtenidos fueron tratados de acuerdo con la Ley de Protección de Datos Personales. El protocolo fue aprobado por el Comité de Evaluación de Protocolos de Investigación del HIB A y la exención de consentimiento informado fue autorizada (Protocolo N. ${ }^{\circ}$ 1999, 17 de enero de 2013).

\section{RESULTADOS}

El estudio comprendió 420 días entre el $12 / 8 / 2013$ y el $5 / 10 / 2014$. En este período, se internaron 732 pacientes, 218 recibieron ARM, se realizaron 1201 observaciones y 10 fueron excluidas por presentar ítems faltantes (ventana de sedación, 3; calentador encendido, 2; alimentación enteral, 2; higiene bucal, 1; transfusión de rojos, 1 ; y $\left.\mathrm{FIO}_{2}<60 \%, 1\right)$. Un total de 1191 observaciones se incluyeron en el estudio. Las características de la población se resumen en la Tabla 1. Durante el estudio, se realizaron 6 evaluaciones periódicas bimestrales. La primera se efectuó al finalizar la prueba piloto, luego de la cual se decidió dicotomizar los ítems de la lista.

De 1191 observaciones incluidas, 183 (15,3\%) resultaron defectuosas, lo que significa que, al menos, una de las prácticas estudiadas no se 
cumplió. En estas 183 unidades de observación, se observó que, en $163(89,1 \%)$, se incumplía 1 práctica; en $18(9,8 \%)$, se incumplían 2 prácticas; y, en $2(1,1 \%)$, se incumplían 3 prácticas.

La evolución del porcentaje de adherencia en el tiempo o proporción de unidades de observación sin defectos se resume en la Figura 1. Esta demuestra una media de $85 \%$ de adherencia y un patrón de variabilidad de causa común que comprende hasta los últimos 8 puntos del gráfico. Estos se ubican por encima de la media y generan un patrón de variabilidad de causa especial que corresponde a los últimos 3 meses del estudio. Las últimas 6 mediciones

TABLA 1. Características de la población estudiada

\begin{tabular}{lc}
\hline Variable & \\
\hline Observaciones, $\mathrm{n}$ & 1191 \\
Pacientes ventilados, $\mathrm{n}$ & 218 \\
Edad en años, mediana (P 25-P 75) & $1,2(0,4-3,3)$ \\
Sexo, varones, \% & 52,7 \\
Peso en kg, mediana (P 25-P 75) & $8,8(5,5-13)$ \\
PIM2, \% ( \pm DE) & $7,8( \pm 13,73)$ \\
Enfermedad de base, \% & 89,6 \\
ARM posquirúrgica, \% & 42,9 \\
Días con ARM, mediana (P 25-P 75) & $4(2-8)$ \\
Mortalidad, \% & 16,5 \\
\hline
\end{tabular}

PIM2: por sus siglas en inglés, Pediatric Index of Mortality 2. ARM: asistencia respiratoria mecánica.

DE: desvío estándar. muestran una adherencia $>90 \%$. La Figura 2 resume bimestralmente la adherencia global y el porcentaje de observaciones defectuosas.

Las prácticas con menor porcentaje de adherencia fueron la interrupción de la sedación y la posición de la cabecera de la cama, con un promedio de incumplimiento de $5,2 \%$ y $4,6 \%$, respectivamente. Las prácticas con mayor porcentaje de adherencia fueron presión plateau $<30 \mathrm{cmH}_{2} \mathrm{O}$, circuito de respirador con menos de 7 días de uso e higiene bucal con un promedio mayor al $99 \%$ y $\mathrm{FIO}_{2}<60 \%$ con $98,2 \%$. La Tabla 2 resume la evolución bimestral de los indicadores de resultado.

\section{DISCUSIÓN}

El uso de la lista de verificación en niños ventilados en la UCIP del HIBA permitió mejorar la adherencia a las prácticas basadas en evidencia estudiadas y alcanzar más de $90 \%$ en los últimos 2 meses del estudio. Dado que el gráfico de control demostró la aparición de variabilidad de causa especial y la lista fue una intervención dirigida a modificar la variabilidad de este proceso, es posible inferir que la mejora alcanzada se asoció a su uso.

El uso de la lista persiguió dos objetivos. Primariamente, constituir una intervención orientada a mejorar la adherencia a las prácticas estudiadas y, de modo secundario, ser una herramienta de registro necesaria para definir

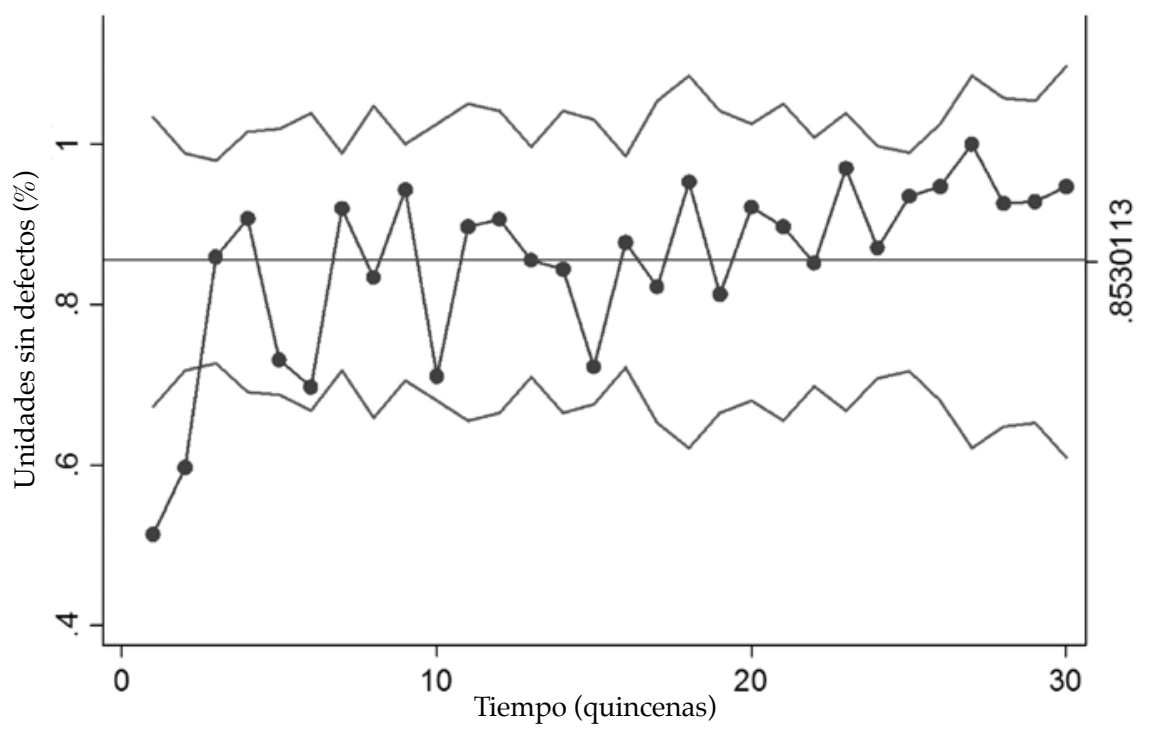

Línea horizontal: media de adherencia.

Línea superior e inferior: límites de control del gráfico a \pm 3 DE. 
variables e indicadores. Solo 4 médicos de planta utilizaron la lista, por ser quienes semanalmente estaban a cargo de la UCIP, lideraban el pase de sala y disponían de privilegios para prescribir el cumplimiento de estas prácticas. El manejo de la lista por este pequeño grupo fue una estrategia pensada desde el diseño para reducir la variabilidad en su uso y, junto con las reuniones bimestrales, mantener el compromiso con su llenado. Weiss demostró que la utilización de listas de cotejo solo mejoraba resultados cuando existía compromiso con su llenado y no cuando se realizaba en forma burocrática. ${ }^{35}$

El gráfico de control demostró variabilidad de causa común en los porcentajes de adherencia durante gran parte del estudio, excepto en 2 ocasiones. La primera fue la correspondiente a las 2 mediciones iniciales, obtenidas durante la prueba piloto, que se ubicaron debajo del límite de control inferior y mostraron una adherencia de alrededor de $50 \%$, los valores más bajos del estudio. Si bien estas mediciones provinieron del período de prueba del instrumento, se decidió incluirlas por lo elocuente de sus valores. Estas demostraron, aunque, tal vez, con menor precisión que las siguientes mediciones, que estos pacientes no se habían beneficiado del conjunto de prácticas estudiadas durante un $50 \%$ de los días que habían permanecido con respirador. Esto contribuyó a describir la magnitud inicial del problema. La segunda ocasión en la que apareció variabilidad de causa especial correspondió a los últimos 3 meses del estudio. Durante ellos, 8 mediciones bisemanales y consecutivas se ubicaron sobre la media. ${ }^{33,34}$ Esto significa que la variabilidad propia de este proceso se alteró, la adherencia aumentó y el cambio sucedido no fue producto del azar.

La evolución simultánea de los indicadores de resultado demostró que, durante el período de estudio, se produjo un descenso en la tasa de neumonías asociadas al respirador, en la de

TABLA 2. Evolución bimestral de los indicadores de resultado

\begin{tabular}{|c|c|c|c|c|c|c|c|c|c|c|}
\hline \multirow[t]{2}{*}{ Indicador } & \multicolumn{3}{|c|}{ PRE } & \multicolumn{7}{|c|}{ POS } \\
\hline & $\begin{array}{c}\text { Mar. } \\
\text { Abr. } 2013\end{array}$ & $\begin{array}{c}\text { May. } \\
\text { Jun. } 2013\end{array}$ & $\begin{array}{c}\text { Jul. } \\
\text { Ago. } 2013\end{array}$ & $\begin{array}{c}\text { Sep. } \\
\text { Oct. } 2013\end{array}$ & $\begin{array}{c}\text { Nov. } \\
\text { Dic. } 2013 \\
\end{array}$ & $\begin{array}{c}\text { Ene. } \\
\text { Feb. } 2014\end{array}$ & $\begin{array}{c}\text { Mar. } \\
\text { Abr. } 2014\end{array}$ & $\begin{array}{c}\text { May. } \\
\text { Jun. } 2014\end{array}$ & $\begin{array}{c}\text { Jul. } \\
\text { Ago. } 2014\end{array}$ & $\begin{array}{c}\text { Sep. } \\
\text { Oct. } 2014\end{array}$ \\
\hline Mortalidad, $\%$ & 5,7 & 3,3 & 4,1 & 4,3 & 4,5 & 5,7 & 4,6 & 6,3 & 5,4 & 5,2 \\
\hline Uso de ARM, \% & 49 & 37 & 29 & 32 & 32 & 43 & 40 & 31 & 39 & 35 \\
\hline Tasa NAR* & 4,9 & 2,9 & 3,9 & 3,3 & 0 & 0 & 7,2 & 0 & 0 & 0 \\
\hline Uso de CVC, \% & 66 & 66 & 67 & 72 & 61 & 62 & 61 & 59 & 52 & 53 \\
\hline Tasa BACVC† & 12,5 & 0 & 6,8 & 6 & 4,1 & 6,6 & 4,7 & 0 & 5.2 & 2,7 \\
\hline Error pres., $\%$ & 1,4 & 3,1 & 1,5 & 1,2 & 1,4 & 1,8 & 1,5 & 2,2 & 1,5 & 1,7 \\
\hline Caídas & 0 & 0 & 0 & 0 & 1 & 0 & 0 & 0 & 0 & 0 \\
\hline
\end{tabular}

ARM: asistencia respiratoria mecánica; NAR: neumonía asociada a respirados; CVC: catéter venoso central;

BACVC: bacteriemia asociada a catéter venoso central; Error pres: errores de prescripción.

* Tasa NAR: episodios de neumonía asociada a respirador por cada 1000 días de uso de asistencia respiratoria mecánica.

† Tasa BACVC: episodios de bacteriemia asociada a catéter venoso central por cada 1000 días de uso de catéter venoso central.

PRE: período de tiempo previo a la intervención; POS: período de tiempo posterior a la intervención.

FIGURA 2. Evolución bimestral de porcentajes de adherencia global y de observaciones defectuosas

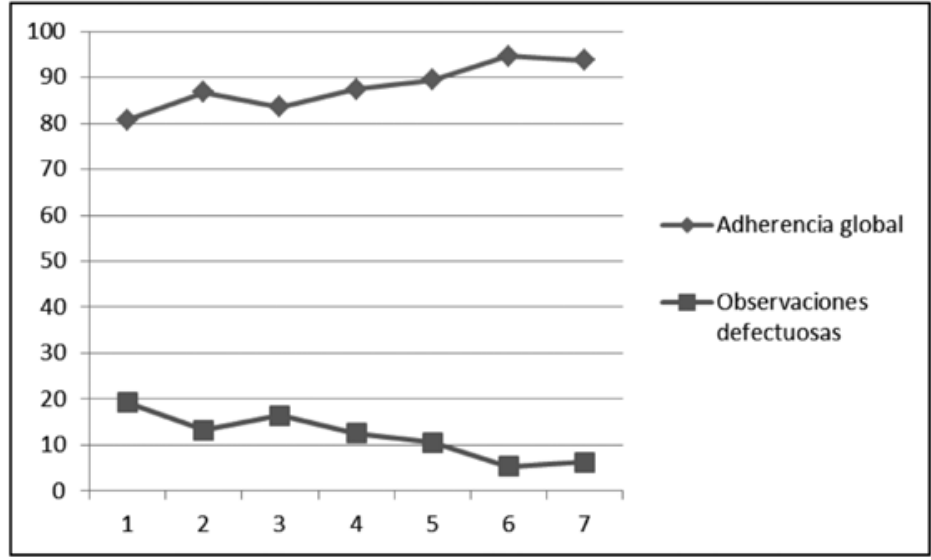


bacteriemias asociadas a catéteres y en la tasa de uso de catéteres venosos centrales. Esta evolución, aunque no es posible afirmar que esté asociada al uso de la lista, permite inferir que los formularios fueron completados en forma comprometida y que es poco probable que hayan existido sesgos al llenarlos.

Existen pocos estudios publicados sobre el uso de listas de cotejo en niños internados en UCIP. Tarrago y cols., reportaron acerca del uso de este instrumento para mejorar la adherencia a prácticas orientadas a mejorar la seguridad de los pacientes durante 21 meses. La lista fue utilizada por un grupo reducido de 5 médicos de planta durante el pase de sala y los autores demostraron mayor adherencia a las prácticas estudiadas y una evolución positiva en indicadores de resultado, como tasa de uso de catéteres, vías arteriales y sondas vesicales, uso de antibióticos endovenosos y número de laboratorios realizados. Estos autores, preocupados porque la lista no fuera llenada en forma burocrática, expusieron ante todo el personal de la UCIP las veces que los médicos habían incumplido su llenado y, además, la evolución de los indicadores registrados. Consideraron que esta estrategia de generar exposición y competencia entre pares había tenido efectos positivos para lograr que la lista se llenara en forma comprometida. ${ }^{17}$ Con características culturales diferentes, aunque con similar objetivo, en este estudio, se realizaron informes periódicos limitados a los médicos que usaron la lista.

Este estudio tiene limitaciones. Por un lado, por haber sido realizado en una UCIP, con características particulares en cuanto a organización y población de pacientes, los resultados obtenidos podrían no ser generalizables. Por otro lado, al no existir un grupo control, no es posible afirmar con certeza que los resultados obtenidos se asocien exclusivamente al uso de la lista. Además, el hecho de no haber estandarizado las justificaciones de cada práctica no realizada podría haber provocado variabilidad en las razones de no adherencia consignadas en la lista. No obstante, al recaer su uso en un grupo reducido de médicos, que compartían el pase de sala y trabajaban juntos desde hacía años, fue una decisión tomada desde el diseño para reducir sesgos relacionados con la falta de estandarización. También podrían haber existido sesgos al registrar motivos de justificación de no adherencia que no correspondieran, lo cual provocaría que se asumieran como cumplidas prácticas que no lo fueron. Esta circunstancia podría haber sobrestimado el porcentaje de adherencia. No obstante, la evolución positiva de algunos indicadores de resultado permite inferir que los resultados reportados son confiables.

Este estudio también tiene fortalezas. La más importante es que se trata de una intervención sencilla de implementar, que no implica gastos excesivos ni riesgos para los pacientes y que, potencialmente, puede mejorar los resultados y la calidad de los cuidados brindados.

\section{CONCLUSIONES}

El uso diario de una lista de cotejo para aumentar la adherencia a prácticas basadas en evidencia (ventana de sedación, estrategias de ventilación con protección pulmonar, prevención de neumonías asociadas a ARM, preferencia de alimentación enteral, disminución del umbral de transfusiones, consideración diaria de prueba de respiración espontánea y retiro del catéter venoso central) permitió mejorar la adherencia al conjunto de estas prácticas y alcanzar más de $90 \%$ en los últimos 2 meses del estudio.

\section{REFERENCIAS}

1. Institute Of Medicine. America's health in transition: protecting and improving quality. Washington DC: National Academy Press; 1994:3-5. [Acceso: 24 de agosto de 2015]. Disponibleen: http: / / www.nap.edu/catalog/9147/ americas-health-in-transition-protecting-and-improvingquality.

2. HesdorfferDC, GhajarJ.Marked improvementinadherence to traumatic brain injury guidelines in United States trauma centers. J Trauma 2007;63(4):841-7.

3. Joynes E, Dalay S, Patel JM, et al. A complete audit cycle to assess adherence to a lung protective ventilation strategy. Indian J Crit Care Med 2014;18(11):746-9.

4. Gurses AP, Seidi KL, Vaidya V, et al. Systems ambiguity and guideline compliance: a qualitative study of how intensive care units follow evidence-based guidelines to reduce healthcare-associated infections. Qual Saf Health Care 2008;17(5):351-9.

5. Pronovost PJ, Rinke ML, Emery K, et al. Interventions to reduce mortality among patients treated in intensive care units. J Crit Care 2004;19(3):158-64.

6. Winters BD, Gurses AP, Lehmann H, et al. Clinical review: Checklists - translating evidence into practice. Crit Care 2009;13(6):210.

7. Young GB, Frewen T, Barr HW, et al. Checklist for diagnosis of brain death. Can J Neurol Sci 1991;18(1):104.

8. Wolff AM, Taylor SA, McCabe JF. Using checklists and reminders in clinical pathways toimprove hospital inpatient care. Med J Aust 2004;181(8):428-31.

9. Haynes AB, Weiser TG, Berry WR, et al. A surgical safety checklist to reduce morbidity and mortality in a global population. N Engl J Med 2009;360(5):491-9.

10. Pronovost P, Berenholtz S, Dorman $\mathrm{T}$, et al. Improving communication in the ICU using daily goals. J Crit Care 2003;18(2):71-5.

11. Berenholtz SM, Milanovich S, Faircloth A, et al. Improving 
care for the ventilated patient. It Comm I Qual Saf 2004;30(4):195-204.

12. Byrnes MC, SchuererDJ, Schallom ME, et al.Implementation of a mandatory checklist of protocols and objectives improves compliance with a wide range of evidence based intensive care unit practices. Crit Care Med 2009;37(10):2775-81.

13. Dubose J, Teixeira PG, Inaba K, et al. Measurable outcomes of quality improvement using a daily quality rounds checklist: one year analysis in a trauma intensive care unit with sustained ventilator associated pneumonia reduction. J Trauma 2010;69(4):855-60.

14. Berenholtz SM, Pronovost PJ, Lipsett PA, et al. Eliminating catheter-related bloodstream infections in the intensive care unit. Crit Care Med 2004;32(10):2014-20.

15. Pronovost P, Needham D, Berenholtz S, et al. An intervention to decrease Catheter Related Bloodstream Infections in the ICU. N Engl J Med 2006;355(26):2725-32.

16. Agarwal S, Frankel L, Tourner $S$, et al. Improving communication in a pediatric intensive care unit using daily patient goal sheets. J Crit Care 2008;23(2):227-35.

17. Tarrago R, Nowak JE, Leonard CS, et al. Reductions in invasive device use and care costs after institution of a daily safety checklist in a pediatric critical care unit. Jt Comm J Qual Patient Saf 2014;40(6):270-8.

18. Gupta K, Gupta VK, Jayashree M, et al. Randomized controlled trial of interrupted versus continuous sedative infusions in ventilated children. Pediatr Crit Care Med 2012;13(2):131-5.

19. KressJP,Pohlman AS, $\mathrm{O}^{\prime}$ ConnorMF, etal. Daily interruption of sedative infusions in critically ill patients undergoing mechanical ventilation. N Engl J Med 2000;342(20):1471-7.

20. PetrucciN, DeFeoC. Lung protective ventilation strategy for the acute respiratory distress syndrome. Cochrane Database Syst Rev 2013;2:CD003844.

21. Curley MA, Schwalenstocker E, Deshpande JK, et al. Tailoring the Institute for Health Care Improvement 100,000 Lives Campaign to pediatric settings: the example of ventilator-associated pneumonia. Pediatr Clin North Am 2006;53(6):1231-51.

22. Bigham MT, Amato R, Bondurrant $\mathrm{P}$, et al. Ventilator associated pneumonia in the pediatric intensive care unit: characterizing the problem and implementing a sustainable solution. J Pediatr 2009;154(4):582-7.e2.

23. Fisher \& Paykel Healthcare. Evaqua Breathing Circuits.
[Acceso: 16 de octubre de 2014]. Disponible en: https:// www.fphcare.co.nz/products/evaqua-circuits.

24. McConville JF, Kress JP. Weaning patients from the ventilator. N Engl J Med 2012;367(23):2233-9.

25. Foronda FK, Troster EJ, Farias JA, et al. The impact of daily evaluation and spontaneous breathing test on the duration of pediatric mechanical ventilation: a randomized controlled trial. Crit Care Med 2011;39(11):2526-33.

26. Ahmed SS, McCaskey MS, Bringman S, et al. Catheter associated bloodstream infection in the pediatric intensive care unit: a multidisciplinary approach. Pediatr Crit Care Med 2012;13(2):e69-72.

27. McClaveSA, MartindaleRG, VanekVW, etal.Guidelines for the provision and assessment of nutrition support therapy in the adult critically ill patient: Society of Critical Care Medicine and American Society for Parenteral and Enteral Nutrition. JPEN J Parenter Enteral Nutr 2009;33(3):277-316.

28. Mehta NM, Bechard LJ, Cahill N, et al. Nutritional practices and their relationship to clinical outcomes in critically ill children: an international multicenter cohort study. Crit Care Med 2012;40(7):2204-11.

29. Lacroix J, Hébert PC, Hutchinson JS, et al. Transfusion strategies for patients in pediatric intensive care units. $N$ Engl J Med 2007;356(16):1609-19.

30. Rouette J, Trottier H, Ducruet T, et al. Red blood cell transfusion threshold in postsurgical pediatric intensive care patients: a randomized clinical trial. Ann Surg 2010;251(3):421-7.

31. Manias E, Williams A, Liew D. Interventions to reduce medication errors in adult intensive care: a systematic review. Br J Clin Pharmacol 2012;4(3):411-23.

32. Pronovost P, Weast B, Schwarz M, et al. Medication reconciliation: a practical tool to reduce the risk of medication errors. J Crit Care 2003;18(4):201-5.

33. Benneyan JC, Lloyd RC, Plsek PE. Statistical process control as a tool for research and healthcare improvement. Qual Saf Health Care 2003;12(6):458-64.

34. Mohammed MA, Worhtington P, Woodall WH. Plotting basic control charts: tutorial notes for healthcare practitioners. Qual Saf Health Care 2008;17(2):137-45.

35. Weiss $\mathrm{CH}$, Moazed F, McEvoy CA, et al. Prompting physicians to address a daily checklist and process of care and clinical outcomes. Am J Respir Crit Care Med 2011;184(6):680-6. 


\section{ANEXO}

Lista de cotejo

\begin{tabular}{|l|l|}
\hline Fecha & \\
\hline Observación número & \\
\hline Apellido del paciente & \\
\hline
\end{tabular}

Lea en voz alta y llene durante el pase de sala de pacientes ventilados

(Excepto traqueostomizados que no reciben sedación endovenosa)

\section{Recibió estándar de cuidado}

\begin{tabular}{|c|c|c|c|}
\hline & si & NO & Por qué* \\
\hline Indica y realiza ventana de sedación & $\mathrm{O}$ & $\mathrm{O}$ & $\cdots$ \\
\hline Realizó ventana de sedación durantelas 24 h previas & $\mathrm{O}$ & $\mathrm{O}$ & 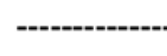 \\
\hline Presión plateau $\leq 30 \mathrm{cmH}_{2} \mathrm{O}$ & $\mathrm{O}$ & $\mathrm{O}$ & \\
\hline $\mathrm{FIO}_{2} \leq 60 \%$ & $\mathrm{O}$ & $\mathrm{O}$ & \\
\hline Calentador humidificador encendido & $\mathrm{O}$ & $\mathrm{O}$ & \\
\hline Tubuladura limpia (véase la fecha de cambio) & $\mathrm{O}$ & $\mathrm{O}$ & \\
\hline Cabecera de la cama a más de $30^{\circ}$ & $\mathrm{O}$ & $\mathrm{O}$ & \\
\hline Indicó higiene bucal & $\mathrm{O}$ & $\mathrm{O}$ & \\
\hline Realizó higiene bucal durante las $24 \mathrm{~h}$ previas & $\mathrm{O}$ & $\mathrm{O}$ & \\
\hline Se planteó prueba de respiración espontânea & $\mathrm{O}$ & $\mathrm{O}$ & \\
\hline Se planteó retirar CVC & $\mathrm{O}$ & $\mathrm{O}$ & \\
\hline Recibió alimentación enteral & $\mathrm{O}$ & $\mathrm{O}$ & \\
\hline Aplicó estándar de transfusión (Hto. $<21 \%$ ) & $\mathrm{O}$ & $\mathrm{O}$ & \\
\hline Reconciliación de medicamentos & $\mathrm{O}$ & $\mathrm{O}$ & \\
\hline
\end{tabular}

* Si no se realizó alguna de estas prácticas, por favor, exprese la razón. 